\title{
Fixed point property for digital spaces
}

\author{
Sang-Eon Han \\ Department of Mathematics Education, Institute of Pure and Applied Mathematics, Chonbuk National University, Jeonju-City Jeonbuk, \\ 561-756, Republic of Korea.
}

Communicated by Y. J. Cho

\begin{abstract}
The paper compares the fixed point property (FPP for short) of a compact Euclidean plane with its digital versions associated with Khalimsky and Marcus-Wyse topology. More precisely, by using a Khalimsky and a Marcus-Wyse topological digitization, the paper studies digital versions of the FPP for Euclidean topological spaces. Besides, motivated by the digital homotopy fixed point property (DHFP for brevity) [O. Ege, I. Karaca, C. R. Math. Acad. Sci. Paris, 353 (2015), 1029-1033], the present paper establishes the digital homotopy almost fixed point property (DHAFP for short) which is more generalized than the DHFP. Moreover, the present paper corrects some errors in [O. Ege, I. Karaca, C. R. Math. Acad. Sci. Paris, 353 (2015), 1029-1033] and improves it. (C) 2017 All rights reserved.
\end{abstract}

Keywords: Digital space, digitization, Khalimsky topology, Marcus-Wyse topology, fixed point property, digital homotopy almost fixed point property, almost fixed point property.

2010 MSC: 55N35, 55M20, 68R10, 68U05.

\section{Introduction}

For a nonempty binary symmetric relation set $(X, \pi)$, we say that $X$ is $\pi$-connected [10] if for any two elements $x$ and $y$ of $X$ there is a finite sequence $\left(x_{i}\right)_{i \in[0, l]_{Z}}$ of elements in $X$ such that $x=x_{0}, y=x_{l}$ and $\left(x_{j}, x_{j+1}\right) \in \pi$ for $j \in[0, l-1]_{\mathbf{Z}}$, where for two distinct integers $a, b \in \mathbf{Z}$ we often use the notation $[a, b]_{\mathbf{Z}}:=\{x \in \mathbf{Z} \mid a \leqslant x \leqslant b\}[14]$. By using this terminology, we say that a digital space is a nonempty, $\pi$-connected, symmetric relation set, denoted by $(X, \pi)[10]$. It is well-known that a digital space [10] includes a digital image $(X, k)$ with digital k-connectivity (i.e., Rosenfeld model) [17, 18], a Khalimsky topological space with Khalimsky adjacency [12], a Marcus-Wyse topological space with Marcus-Wyse adjacency [22] and so forth [15]. Besides, a digital space can be established in terms of a digitization of a Euclidean space [11]. Thus the paper deals with both the fixed point property (FPP for short) for Euclidean topological spaces in $\mathbf{R}^{n}$ and the FPP for their digitized spaces in $\mathbf{Z}^{n}$ from the viewpoint of digital topology. We say that a digital image $(X, k)$ is $k$-connected if it is not a union of two disjoint non-empty sets that are not $k$-adjacent to each other [14]. At this moment we need to recall that the digital image $(X, k)$ on $Z^{n}$ is not a topological space but just a digital graph on $\mathbf{Z}^{\mathfrak{n}}$ with a k-adjacency. We say that a non-empty and $k$-connected digital image $(X, k)$ has the FPP [18] if every $k$-continuous map

Email address: sehan@jbnu .ac.kr (Sang-Eon Han)

doi:10.22436/jnsa.010.05.20

Received 2017-01-19 
$f:(X, k) \rightarrow(X, k)$ has a point $x \in X$ such that $f(x)=x$. In fixed point theory from the viewpoint of digital topology in a graph-theoretical approach, we also assume that every digital image $(X, k)$ is $k$-connected and non-empty.

To compare the FPP for Euclidean topological spaces with its digital version associated with Rosenfeld, Khalimsky, and Marcus-Wyse model, let us recall basic notions on fixed point theory and digital topology. Hereafter, we use the simplified words ' $\mathrm{K}-$ ' and 'M-' instead of Khalimsky, and Marcus-Wyse, respectively if there is no danger of ambiguity. In digital topology, since there are several kinds of digitizations [11], Sections 2 and 4 follow the graph-theoretical approach in [17, 18] and Section 3 mainly deals with digitizations associated with a K- and an M-topological structure. Besides, we recall that under some conditions, Euler characteristics of digital images can be used to study the FPP for digital images. We say that a non-empty digital image $(X, k)$ has the almost fixed point property (AFPP, for brevity) [18] if every $k$-continuous map $f:(X, k) \rightarrow(X, k)$ has a point $x \in X$ such that $f(x)=x$ or $f(x)$ is k-adjacent to $x$ [18]. It is obvious that the AFPP is broader than the FPP [18].

We say that a topological space $X$ has the homotopy fixed point property (HFP for short) [21] if it has the FPP with respect to the unit interval $[0,1]$. Motivated by the HFP, the authors of [3] recently established its digital version named by the digital homotopy fixed point property (DHFP for short) in terms of a (digitally) kcontinuous map [18], adjacency relations of Cartesian products of digital images [4], a digital k-homotopy [2] and so forth. This approach can contribute to topology and applied sciences. Besides, we say that a digital image $(\mathrm{X}, \mathrm{k})$ has the DHFP [3] if it has the digital fixed point property (DFP for brevity) with respect to any integral interval $[0, \mathrm{~m}]_{\mathbf{Z}} \subset \mathbf{Z}$ (see Definition 4.7 of the present paper). It is well known that only a singleton has the FPP [18]. Although the authors of [3] studied the DHFP in terms of a graph-theoretical approach, the work can be simplified as one statement (see Proposition 1.1 below) because it is wellknown that a digital image $(X, k)$ does not have the FPP with $|X| \geqslant 2$ (see Theorems 3.3 and 4.1 of [18] and the papers $[6,7])$. Namely, only a singleton has the FPP and further, the DHFP of $(\mathrm{X}, \mathrm{k})$ requires its digital fixed point property (DFP for short) (see Corollary 3.3 of [3]). Hence we clearly obtain the following:

Proposition 1.1. A digital image $(X, k)$ has the DHFP if and only if it is a singleton.

Since the paper [3] has some errors, the present paper corrects them and makes the paper improved. Furthermore, based on Proposition 1.1, the present paper proposes the 'digital homotopy almost fixed point property (DHAFP for short)' which is weaker than the DHFP in [3].

The rest of the paper is organized as follows: Section 2 provides basic notions from digital topology. Section 3 investigates some properties of digitizations in a K- and an $\mathrm{M}$-topological approach and further, studies some relations between the $F P P$ for spaces in $\mathrm{R}^{\mathrm{n}}$ and its digital versions. Furthermore, it refers to the FPP for $\mathrm{K}$ - and M-topological spaces. Section 4 studies the DHFP. Section 5 investigates a nonhomotopy equivalence property of the DHFP. Section 6 deals with the almost fixed point property of digital images and studies various properties of the DHAFP. Besides, we prove that both a bounded simple k-path in $\mathbf{Z}^{\mathrm{n}}$ and a bounded digital plane with 8-adjacency satisfy the DHAFP.

\section{Preliminaries}

To study the FPP for digital spaces and the AFPP for digital images from the viewpoint of digital topology, we need to recall some basic notions from digital topology such as k-adjacency relations of $\mathrm{nD}$ integer grids, a digital k-neighborhood, digital continuity and so forth $[5,17,18]$. Let $\mathbf{N}, \mathbf{Z}^{\mathrm{n}}$, and $\mathbf{R}$ represent the sets of natural numbers, points in the Euclidean $\mathrm{nD}$ space with integer coordinates, and real numbers, respectively.

To study $n D$ digital images, $n \in \mathbf{N}$, as a generalization of the k-adjacency relations of $\mathbf{Z}^{n}, n \in\{1,2,3\}$, we will take the following property [5].

For a natural number $m, 1 \leqslant m \leqslant n$, two distinct points

$$
p=\left(p_{1}, p_{2}, \cdots, p_{n}\right) \text { and } q=\left(q_{1}, q_{2}, \cdots, q_{n}\right) \in \mathbf{Z}^{n} \text {, }
$$


are $k(m, n)$-adjacent if

at most $m$ of their coordinates differ by \pm 1 , and all others coincide.

In terms of the operator of (2.1), the $k(m, n)$-adjacency relations of $\mathbf{Z}^{n}, n \in \mathbf{N}$, are obtained [5] as follows:

$$
k:=k(m, n)=\sum_{i=n-m}^{n-1} 2^{n-i} C_{i}^{n}, \text { where } C_{i}^{n}=\frac{n !}{(n-i) ! i !} .
$$

Rosenfeld [17] called a set $X\left(\subset Z^{n}\right)$ with a k-adjacency a digital image, denoted by $(X, k)$. Indeed, to study digital images on $\mathbf{Z}^{\mathrm{n}}$ in the graph-theoretical approach [17, 18], using the k-adjacency relations of $\mathbf{Z}^{n}$ of (2.2), we say that a digital k-neighborhood of $p$ in $\mathbf{Z}^{n}$ is the set [17] $N_{k}(p):=$ $\{q \mid p$ is $k$-adjacent to $q\} \cup\{p\}$. Furthermore, we often use the notation [13] $N_{k}^{*}(p):=N_{k}(p) \backslash\{p\}$. For $a, b \in \mathbf{Z}$ with $a \lessgtr b$, the set $[a, b]_{Z}=\{m \in Z \mid a \leqslant m \leqslant b\}$ with 2-adjacency is called a digital interval. Besides, for a k-adjacency relation of $\mathbf{Z}^{n}$, a simple k-path with $l+1$ elements in $\mathbf{Z}^{\mathrm{n}}$ is assumed to be a subset $\left(x_{i}\right)_{i \in[0, l]_{z}} \subset \mathbf{Z}^{n}$ such that $x_{i}$ and $x_{j}$ are $k$-adjacent if and only if $|i-j|=1$. If $x_{0}=x$ and $x_{l}=y$, then the length of the simple $k$-path, denoted by $l_{k}(x, y)$, is the number $l$. A simple closed $k$-curve with $l$ elements in $\mathbf{Z}^{\mathrm{n}}$, denoted by $S C_{k}^{n, l}$ [5], is the simple k-path $\left(x_{i}\right)_{i \in[0, l-1]_{z}}$, where $x_{i}$ and $x_{j}$ are $k$-adjacent if and only if $|\mathfrak{i}-\mathfrak{j}|=1(\bmod \boldsymbol{l})$.

For a digital image $(X, k)$, for $X \subset Z^{n}$ we put [5]

$$
\mathrm{N}_{\mathrm{k}}(x, 1):=\mathrm{N}_{\mathrm{k}}(x) \cap X .
$$

As a generalization of $N_{k}(x, 1)$ of (2.3), for a digital image $(X, k)$ let us recall a digital $k$-neighborhood [5]. Namely, the digital $k$-neighborhood of $x_{0} \in X$ with radius $\varepsilon$ is defined in $X$ to be the following subset of $\mathrm{X}[5]$

$$
N_{k}\left(x_{0}, \varepsilon\right):=\left\{x \in X \mid l_{k}\left(x_{0}, x\right) \leqslant \varepsilon\right\} \cup\left\{x_{0}\right\},
$$

where $l_{k}\left(x_{0}, x\right)$ is the length of a shortest simple k-path from $x_{0}$ to $x$ and $\varepsilon \in \mathbf{N}$.

Definition 2.1. We say that a $k$-connected digital image $(X, k)$ on $Z^{n}$ is bounded if for some point $x_{0} \in X$ there is an $N_{k}\left(x_{0}, \varepsilon\right)$ such that $X=N_{k}\left(x_{0}, \varepsilon\right)$, where $\varepsilon \lesseqgtr \infty$.

For two digital images $\left(X, k_{1}\right)$ in $Z^{n_{1}}$ and $\left(Y, k_{2}\right)$ in $Z^{n_{2}}$, we have often used the following adjacency for a digital product $X \times Y=\{(x, y) \mid x \in X, y \in Y\} \subset Z^{n_{1}+n_{2}}$, as follows.

Definition 2.2 ([5]). For two digital images $\left(X, k_{1}\right)$ in $Z^{n_{1}},\left(Y, k_{2}\right)$ in $Z^{n_{2}}$, consider the digital product $X \times Y \subset Z^{n_{1}+n_{2}}$. Then we say that two points $(x, y) \in X \times Y,\left(x^{\prime}, y^{\prime}\right) \in X \times Y$ are normally k-adjacent to each other if and only if

(1) $x$ is $k_{1}$-adjacent to $x^{\prime}$ and $y=y^{\prime}$; or

(2) $y$ is $k_{2}$-adjacent to $y^{\prime}$ and $x=x^{\prime}$; or

(3) $x$ is $k_{1}$-adjacent to $x^{\prime}$ and $y$ is $k_{2}$-adjacent to $y^{\prime}$.

The paper [18] established the notion of digital continuity of a map $f:\left(X, k_{0}\right) \rightarrow\left(Y, k_{1}\right)$ by saying that $f$ maps every $k_{0}$-connected subset of $\left(X, k_{0}\right)$ into a $k_{1}$-connected subset of $\left(Y, k_{1}\right)$ (see Theorem 2.4 of $[18]$ ). Motivated by this approach, the digital continuity of maps between digital images was represented in terms of the neighborhood of (2.3), as follows:

Proposition 2.3 ([5]). Let $\left(X, k_{0}\right)$ and $\left(Y, k_{1}\right)$ be digital images in $\mathbf{Z}^{n_{0}}$ and $\mathbf{Z}^{n_{1}}$, respectively. A function $f$ : $\left(\mathrm{X}, \mathrm{k}_{0}\right) \rightarrow\left(\mathrm{Y}, \mathrm{k}_{1}\right)$ is $\left(\mathrm{k}_{0}, \mathrm{k}_{1}\right)$-continuous if and only if for every $\mathrm{x} \in \mathrm{X}, \mathrm{f}\left(\mathrm{N}_{\mathrm{k}_{0}}(\mathrm{x}, 1)\right) \subset \mathrm{N}_{\mathrm{k}_{1}}(\mathrm{f}(\mathrm{x}), 1)$.

Definition 2.4 ([2] (see also [9])). Consider two digital images $\left(X, k_{0}\right)$ and $\left(Y, k_{1}\right)$ in $Z^{n_{0}}$ and $Z^{n_{1}}$, respectively. Then a map $h: X \rightarrow Y$ is called a $\left(k_{0}, k_{1}\right)$-isomorphism if $h$ is a $\left(k_{0}, k_{1}\right)$-continuous bijection and further, $h^{-1}: Y \rightarrow X$ is $\left(k_{1}, k_{0}\right)$-continuous. 


\section{Comparison the FPP of a compact Euclidean plane with its digital versions}

Motivated by an Alexandroff space [1], the Khalimsky nD space was established and the study of its properties includes the papers [11-13]. More precisely, Khalimsky line topology $\mathrm{K}$ on $\mathbf{Z}$, denoted by $(\mathbf{Z}, \mathrm{k})$, is induced by the set $\left\{[2 n-1,2 n+1]_{\mathbf{Z}}: \mathfrak{n} \in \mathbf{Z}\right\}$ as a subbase [12] (see also [13]). Furthermore, the product topology on $\mathbf{Z}^{\mathrm{n}}$ induced by $(\mathbf{Z}, \mathbf{k})$ is called the Khalimsky product topology on $\mathbf{Z}^{\mathrm{n}}$ (or the Khalimsky $\mathrm{n} D$ space), denoted by $\left(\mathbf{Z}^{\mathfrak{n}}, \mathrm{k}^{\mathrm{n}}\right)$. For the sake of convenience, we say that a point $\mathrm{x}=\left(\mathrm{x}_{1}, \mathrm{x}_{2}, \cdots, \mathrm{x}_{\mathrm{n}}\right) \in \mathbf{Z}^{\mathrm{n}}$ is pure open if all coordinates are odd; and it is pure closed if each of the coordinates is even [13]. The other points in $\mathbf{Z}^{n}$ are called mixed [13]. In $\mathbf{Z}^{2}$, these points are showed like Figures 2-4, the symbols $\mathbf{\square}$, a black big circle, $\bullet$ mean a pure closed point, a pure open point, and a mixed point, respectively.

As usual, for a subset $X \subset \mathbf{Z}^{n}$ we will consider $\left(X, \kappa_{X}^{n}\right), n \geqslant 1$ [7] as a subspace of $\left(Z^{n}, \kappa^{n}\right)$, and it is called a K-topological space. For two K-topological spaces $\left(X, \kappa_{X}^{n_{0}}\right):=X$ and $\left(Y, K_{Y}^{n_{1}}\right):=Y$ we say that a map $f: X \rightarrow Y$ is $K$-continuous if it is K-continuous at every point $x \in X$, i.e, $f\left(\operatorname{SN}_{K}(x)\right) \subset S N_{K}(f(x))$ $[7,8,11]$, where $S N_{K}(x)$ is the smallest open set containing the point $x$ from the viewpoint of Khalimsky topology. By using spaces $\left(X, \kappa_{X}^{n}\right):=X$ and $K$-continuous maps, we have a Khalimsky topological category, denoted by KTC [11], consisting of the following two sets [7]:

(1) for any set $X \subset Z^{n}$, the set of spaces $\left(X, \kappa_{X}^{n}\right)$ as objects of $K T C$ denoted by $O b(K T C)$;

(2) for all pairs of elements in $\mathrm{Ob}(\mathrm{KTC})$ the set of all $\mathrm{K}$-continuous maps between them as morphisms.

The paper [7] studied the FPP for K-topological spaces and further, the paper [7] proved that not every $\mathrm{K}$-contractible space does not have the FPP.

Let us now recall basic concepts from Marcus-Wyse (M-, for short) topology as another digital space. The M-topology on $\mathbf{Z}^{2}$, denoted by $\left(\mathbf{Z}^{2}, \gamma\right)$, is induced by the set $\{U\}$ in (3.1) below as a subbase [22], where for each point $p=(x, y) \in \mathbf{Z}^{2}$

$$
\mathrm{U}:=\mathrm{SN}_{\mathrm{M}}(\mathrm{p}):=\mathrm{N}_{4}(\mathrm{p}) \text { if } x+y \text { is even. }
$$

In relation to the further statement of a point in $\mathbf{Z}^{2}$, in the paper we call a point $p=\left(x_{1}, x_{2}\right)$ double even if $x_{1}+x_{2}$ is an even number such that each $x_{i}$ is even, $i \in\{1,2\}$; even if $x_{1}+x_{2}$ is an even number such that each $x_{i}$ is odd, $i \in\{1,2\}$; and odd if $x_{1}+x_{2}$ is an odd number [20].

In all subspaces of $\left(\mathbf{Z}^{2}, \gamma\right)$ of Figures 2-4, a black jumbo dot means an even point and further, the symbol $\diamond$ means a double even point or a even point, and the symbol $\bullet$ means an odd point. In view of (3.1), we can obviously obtain the following: under $\left(\mathbf{Z}^{2}, \gamma\right)$ the singleton with either a double even point or an even point is the closure containing the given point. In addition, the singleton with an odd point is clearly the smallest open neighborhood of the given point. For a set $X \subset Z^{2}$ we can take the subspace, denoted by $\left(X, \gamma_{X}\right)$, induced by $\left(Z^{2}, \gamma\right)$. As usual, for a subset $X \subset Z^{2}$ we will consider $\left(X, \gamma_{X}^{2}\right)$ [22] as a subspace of $\left(\mathbf{Z}^{2}, \gamma^{2}\right)$, and it is called an $M$-topological space. For two $M$-topological spaces $\left(X, \gamma_{X}\right):=X$ and $\left(Y, \gamma_{Y}\right):=Y$, a function $f: X \rightarrow Y$ is said to be $M$-continuous at a point $x \in X$ [22], if $f\left(S N_{M}(x)\right) \subset S N_{M}(f(x))$ [11], where $S N_{M}(x)$ is the smallest open set containing the point $x$ from the viewpoint of $M$-topology. Besides, a map $f: X \rightarrow Y$ is $M$-continuous if it is $M$-continuous at every point $x \in X$. By using spaces $\left(X, \gamma_{X}\right):=X$ and $M$-continuous maps, we have an $M$-topological category, denoted by $M T C$, consisting of the following two sets [11]:

(1) for any set $X \subset \mathbf{Z}^{2}$, the set of spaces $\left(X, \gamma_{X}\right)$ as objects of $M T C$ denoted by $O b(M T C)$;

(2) for all pairs of elements in $\mathrm{Ob}(\mathrm{MTC})$ the set of all M-continuous maps between them as morphisms.

To compare the FPP for Euclidean spaces with its digital version, let us investigate two digitizations such as a K- and an M-digitization. Given a Euclidean space $\mathrm{X} \subset \mathbf{R}^{\mathrm{n}}, \mathrm{K}$-topological and $\mathrm{M}$-topological digitizations were introduced in [11].

The following local K-neighborhood of a point $p \in \mathbf{Z}^{\mathrm{n}}$ plays an important role in $\mathrm{K}$-digitizing $\mathrm{X} \subset \mathbf{R}^{\mathrm{n}}$. 
Definition 3.1 ([8]). In $\mathbf{R}^{n}$, for each point $p:=\left(p_{i}\right)_{i \in[1, n]_{Z}} \in \mathbf{Z}^{n}$, we define the set associated with $\left(\mathbf{Z}^{n}, k^{n}\right)$, as follows:

$$
N_{K}(p):=\left\{\left(x_{i}\right)_{i \in[1, n]_{z}}\right\}, \text { where }\left\{\begin{array}{l}
\text { if } p_{i}=2 m, \text { then } x_{i} \in\left[2 m-\frac{1}{2}, 2 m+\frac{1}{2}\right], \\
\text { if } p_{i}=2 m+1, \text { then } x_{i} \in\left(2 m+\frac{1}{2}, 2 m+\frac{3}{2}\right),
\end{array}\right\},
$$

which is called a local K-neighborhood associated with $\left(\mathbf{Z}^{\mathrm{n}}, \mathrm{k}^{\mathrm{n}}\right)$.

For instance, we can observe $N_{K}(p)$ in the Euclidean 3-dimensional space (see Figures 1-4).

Hereafter, we denote by $\left(\mathbf{R}^{n}, E^{n}\right)$ the Euclidean $n$-dimensional space.

Definition 3.2 ([8]). For two points $x, y \in\left(\mathbf{R}^{n}, E^{n}\right), x$ is related to $y$ if $x, y \in N_{K}(p)$ for some point $p \in \mathbf{Z}^{n}$, denoted by $x \sim_{k} y$ which is an equivalence relation.

By using this approach, we may consider $\bigcup_{p \in Z^{n}} N_{K}(p)$ as $\mathbf{R}^{n}$ which is used to digitize $\left(\mathbf{R}^{n}, E^{n}\right)$ into $\left(Z^{n}, K^{n}\right)$. Besides, it is clear that for two points $p, q$ in $Z^{n}$ with $p \neq q, N_{K}(p) \cap N_{K}(q)=\emptyset$ so that the set $\left\{N_{K}(p) \mid p \in Z^{n}\right\}$ is a partition of $\mathbf{R}^{n}[11]$.

Definition $3.3([11])$. Let $X$ be a subspace in $\left(R^{n}, E^{n}\right)$. A K-digitization of $X$, denoted by $D_{K}(X)$, is defined as follows:

$$
\mathrm{D}_{\mathrm{K}}(\mathrm{X})=\left\{\mathrm{p} \in \mathbf{Z}^{\mathrm{n}} \mid \mathrm{N}_{\mathrm{K}}(\mathrm{p}) \cap \mathrm{X} \neq \emptyset\right\}
$$

with K-topology.

Example 3.4. Let us consider the curve $X$ in Figure 2 (a). After K-digitizing $X$, we obtain the set $D_{K}(X):=$ $\{(1,0),(1,1),(2,1),(3,1),(4,1),(4,2)\}$. In this case the singletons $\{(1,1)\}$ and $\{(3,1)\}$ are the smallest open sets containing the given points, respectively. Besides, the singleton $\{(4,2)\}$ is the closed set.

Let us now consider a digitization of a Euclidean 2D subspace $X \subset \mathbf{R}^{2}$ in the Marcus-Wyse (for brevity, M-) topological approach. The following local M-neighborhood of a point $p \in \mathbf{Z}^{2}$ plays an important role in M-digitizing $X \subset \mathbf{R}^{2}$, as follows:

Definition 3.5 ([11]). In $\mathbf{R}^{2}$, for each point $p \in \mathbf{Z}^{2}$, we define the following neighborhood of $p$ (see Figures 1 (b), (5)-(7)): for $i \in\{1,2\}$

$$
N_{M}(p):=\left\{\begin{array}{l}
\left\{\left(t_{1}, t_{2}\right) \mid t_{i} \in\left[p_{i}-\frac{1}{2}, p_{i}+\frac{1}{2}\right]\right\} \text { if } p=\left(p_{1}, p_{2}\right) \text { is a double even point, and } \\
\left\{\left(t_{1}, t_{2}\right) \mid t_{i} \in\left[p_{i}-\frac{1}{2}, p_{i}+\frac{1}{2}\right]\right\} \backslash\left\{\left(p_{1} \pm \frac{1}{2}, p_{2} \pm \frac{1}{2}\right)\right\} \text { if } p=\left(p_{1}, p_{2}\right) \text { is an even point, and } \\
\left\{\left(t_{1}, t_{2}\right) \mid t_{i} \in\left(p_{i}-\frac{1}{2}, p_{i}+\frac{1}{2}\right)\right\} \text { if } p=\left(p_{1}, p_{2}\right) \text { is an odd point }
\end{array}\right\},
$$

which is called the local M-neighborhood of $p$ associated with $\left(\mathbf{Z}^{2}, \gamma\right)$.

Definition 3.6 ([11]). For two points $x, y \in\left(\mathbf{R}^{2}, E^{2}\right)$, we say that $x$ is related to $y$ if $x, y \in N_{M}(p)$ for some point $p \in \mathbf{Z}^{2}$, denoted by $x \sim_{M} y$ which is an equivalence relation.

It is clear that the set $\left\{N_{M}(p) \mid p \in \mathbf{Z}^{2}\right\}$ is a partition of $\mathbf{R}^{2}$ associated with M-topology [11].

Definition 3.7 ([11]). Let $X$ be a subspace in $\left(\mathbf{R}^{2}, E^{2}\right)$. An $M$-digitization $D_{M}(X)$ of $X$ is defined as follows:

$$
\mathrm{D}_{\mathrm{M}}(\mathrm{X})=\left\{\mathrm{p} \in \mathbf{Z}^{2} \mid \mathrm{N}_{\mathrm{M}}(\mathrm{p}) \cap \mathrm{X} \neq \emptyset\right\}
$$

with M-topology. 

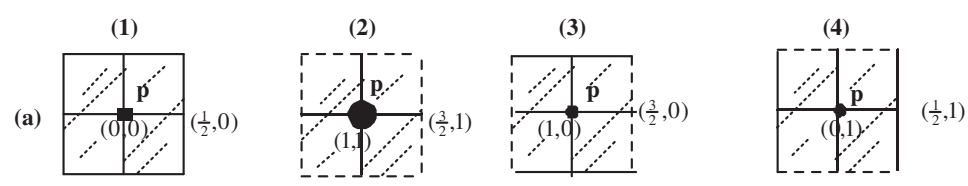

$\mathbf{N}_{\mathbf{K}}(\mathbf{p})$ corresponding to the pure closed, pure open, mixed points $p$

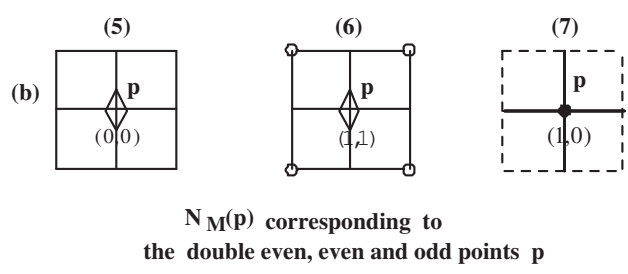

Figure 1: (a) Local K-neighborhoods of given points $p$ in (1)-(4); (b) Local M-neighborhoods of given points $p$ in (5)-(7) [11].

Example 3.8. Let us consider the curve $X$ in Figure $2(b)$. After $M$-digitizing $X$, we obtain the set $D_{M}(X):=$ $\{(1,0),(1,1),(2,1),(3,1),(4,1),(4,2)\}$. In this case each of the singletons $\{(1,0)\},\{(2,1)\}$, and $\{(4,1)\}$ is the smallest open set containing the given point. Besides, each of the singletons $\{(1,1)\},\{(3,1)\}$, and $\{(4,2)\}$ is a closed set.

Remark 3.9. In view of Examples 3.4 and 3.8, we see that $D_{K}(X)$ and $D_{M}(X)$ have quite different topological structures.

For a closed interval $X \subset \mathbf{R}$ and an open interval $Z \subset \mathbf{R}$, owing to Brouwer fixed point theorem [16], in Euclidean topology it is clear that whereas $X$ has the FPP, $Y$ does not have the FPP. Thus we have a query of a relation between the FPP of $\mathrm{X}$ and that of its $\mathrm{K}$ - and $\mathrm{M}$-digitizations, as follows:

Remark 3.10. Let $X:=\{x \in \mathbf{R}: a \leqslant x \leqslant b, a, b \in \mathbf{Z}\}$ be a closed interval (see Figure 2 (c)), $Y:=\{y \in \mathbf{R}: a \leqslant$ $\mathrm{y} \lesseqgtr \mathrm{b}, \mathrm{a}, \mathrm{b} \in \mathbf{Z}\}$ be a closed-open interval (see Figure 2 (c)), and $\mathbf{Z}:=\{z \in \mathbf{R}: \mathrm{a} \lessgtr z \lessgtr \mathrm{b}, \mathrm{a}, \mathrm{b} \in \mathbf{Z}\}$ be an open interval. Then we obtain the followings:

(1) $\mathrm{D}_{\mathrm{K}}(\mathrm{X})=\mathrm{D}_{\mathrm{K}}(\mathrm{Y})=\mathrm{D}_{\mathrm{K}}(\mathrm{Z})$

(2) Each of $D_{K}(X), D_{K}(Y)$, and $D_{K}(Z)$ has the FPP in $K T C$.

(3) $\mathrm{D}_{M}(\mathrm{X})=\mathrm{D}_{\mathrm{M}}(\mathrm{Y})=\mathrm{D}_{\mathrm{M}}(\mathrm{Z})$.

(4) Each of $D_{M}(X), D_{M}(Y)$, and $D_{M}(Z)$ has the FPP in MTC.

Indeed, according to Definitions 3.3 and 3.7, since each of $D_{K}(X), D_{K}(Y)$, and $D_{K}(Z)$ is a K-path as a same set, it was proved to have the $F P P$ in $K T C[7,19]$. Besides, each of $D_{M}(X), D_{M}(Y)$, and $D_{M}(Z)$ is, respectively, equivalent to each of $D_{K}(X), D_{K}(Y)$, and $D_{K}(Z)$ up to $K$ - or $M$-homeomorphism. In addition, each of $D_{M}(X), D_{M}(Y)$, and $D_{M}(Z)$ also has the FPP in MTC because the FPP is a topological invariant property [7].

Theorem 3.11. A compact M-topological plane does not have the FPP.

Proof. It suffices to prove the assertion with a counterexample. Let us consider the M-topological space $\left(X, \gamma_{X}\right)$ suggested in Figure 3, i.e., $X:=\left\{x_{i}, y_{i}, z_{j}, w_{j} \mid i \in[0,5]_{Z}, j \in[0,1]_{Z}\right\}$. Assume the self-map $f$ of $\left(X, \gamma_{X}\right)$ given by

$$
f\left(x_{i}\right)=y_{i}, f\left(y_{i}\right)=x_{i}, f\left(z_{j}\right)=w_{j}, f\left(w_{j}\right)=z_{j}, i \in[0,5]_{\mathbf{Z}}, j \in[0,1]_{\mathbf{Z}},
$$

which is a rotation of $X$ by $180^{\circ}$. Then it is clear that whereas $f$ is an M-continuous map, $f$ cannot have any fixed point, which implies that $\left(X, \gamma_{X}\right)$ does not have the FPP. 

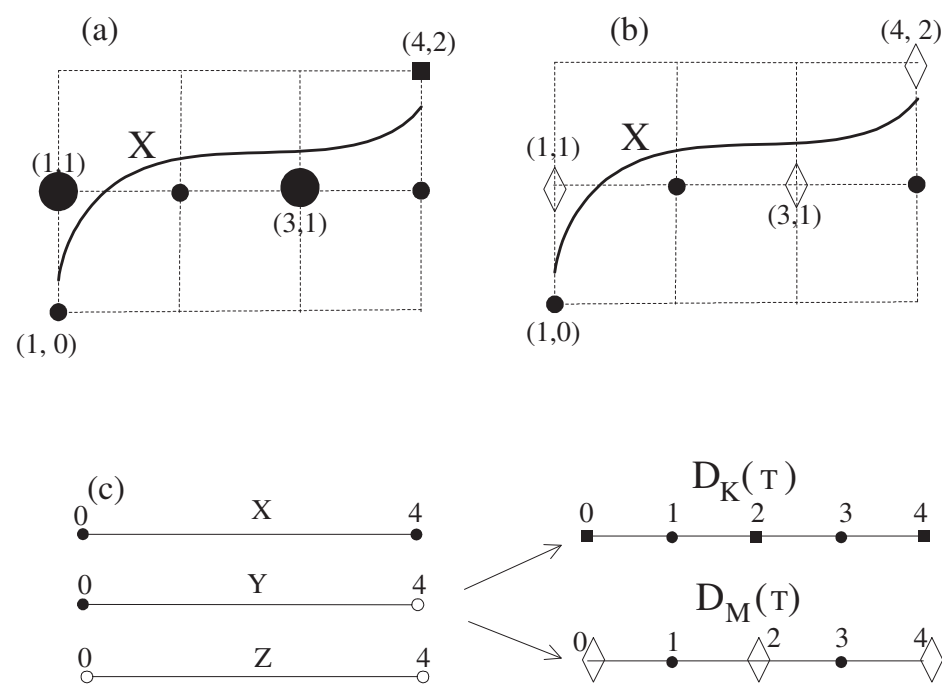

Figure 2: (a) A K-digitization of the given curve $X \subset \mathbf{R}^{2}$; (b) An M-digitization of the given curve $X \subset \mathbf{R}^{2}$; (c) A K- and an $M$-digitization of $T \subset \mathbf{R}$, where $T \in\{X, Y, Z\}, X:=[0,4], Y:=[0,4), Z:=(0,4)$.
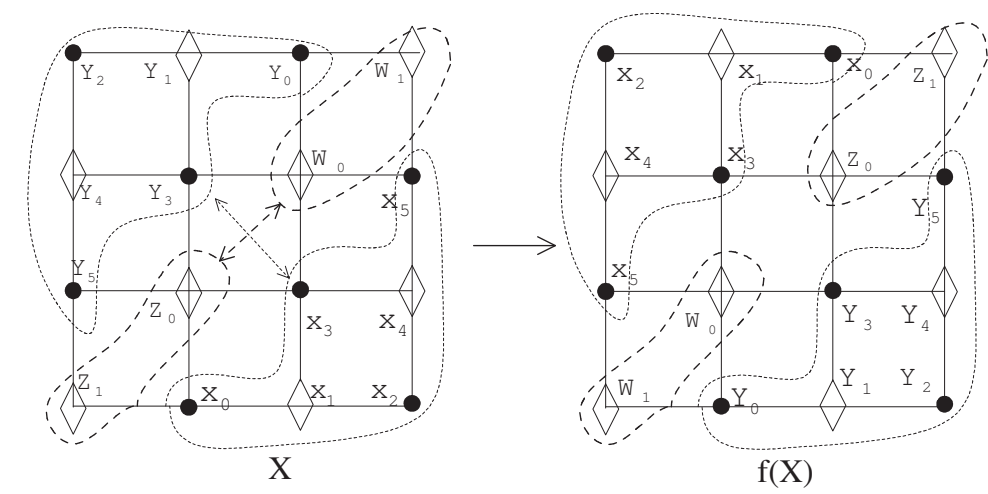

Figure 3: Explanation of the non-FPP of a compact M-topological plane.

Based on the above assertions, we will compare the FPP among a Euclidean, a K-, and an M-plane as follows:

Proposition 3.12. For a compact Euclidean topological plane $[-1,1] \times[-1,1]:=\mathrm{X}$, whereas its $\mathrm{K}$-digitized plane $\left(\mathrm{D}_{\mathrm{K}}(\mathrm{X}), \mathrm{\kappa}_{\mathrm{D}_{\mathrm{K}}(\mathrm{X})}^{2}\right)$ has the FPP, its M-digitized plane $\left(\mathrm{D}_{\mathrm{M}}(\mathrm{X}), \gamma_{\mathrm{D}_{\mathrm{M}}(\mathrm{X})}\right)$ does not have the FPP.

Proof. It is clear that a compact Euclidean plane $X:=[-1,1] \times[-1,1]$ has the FPP [16].

Let us now prove that whereas its $K$-digitized space $\left(D_{K}(X), \kappa_{D_{K}(X)}^{2}\right)$ has the FPP, its M-digitized space $\left(\mathrm{X}, \gamma_{\mathrm{X}}\right)$ does not have the FPP.

Case 1: The FPP of $\left(D_{K}(X), \kappa_{D_{K}(X)}^{2}\right)$. Let us consider any K-continuous self-map of $D_{K}(X)$. For convenience, take $x:=(0,0)$ as a pure closed point.

(1-1) If $x$ is mapped by the map $f$ into $x$, then the proof is obviously completed.

(1-2) We may assume that $x$ is mapped into one of the mixed points by the map $f$ such as $x_{1}$, where $x_{1}$ is a mixed point of $D_{K}(X)$. 
For convenience, put $x_{1}:=(0,1)$ (see Figure $4(\mathrm{a})$ ). Owing to the K-topological structures of $\mathrm{SN}_{\mathrm{K}}(\mathrm{x})$ and $S N_{K}\left(x_{1}\right)$, it is clear that $f\left(S N_{K}(x)\right) \subset S N_{K}\left(x_{1}\right)=\left\{(-1,1), x_{1},(1,1)\right\}$. Then we need to consider the mapping $f$ focused on $S N_{K}\left(x_{1}\right)$, as follows:

In case $f\left(x_{1}\right)=x_{1}$, the proof is completed.

In case $f\left(x_{1}\right)=i \in\{(-1,1),(1,1)\}$, we should have $f(i)=i$ because $S N_{K}(i)=\{i\}$, which completes the proof.

(1-3) Assume that $x$ is mapped by the map $f$ into one of the pure open points of $D_{K}(X)$ such as $x_{2}$, where $x_{2}$ is a pure open point in $S N_{K}(x)$. Then, owing to the K-topological structure of $S N_{K}\left(x_{2}\right)$, we obviously obtain $f\left(S N_{K}(x)\right) \subset S N_{K}\left(x_{2}\right)=\left\{x_{2}\right\}$. Hence we obtain the point $x_{2}$ as a fixed point of $f$.

Similarly, the other cases are proved for the FPP of $\mathrm{D}_{\mathrm{K}}(\mathrm{X})$. Thus a compact $\mathrm{K}$-plane $\mathrm{D}_{\mathrm{K}}(\mathrm{X})$ is proved to have the FPP.

Case 2: The non-FPP of $\left(D_{M}(X), \gamma_{D_{M}(X)}\right)$. Let us now prove the non-FPP of $\left(D_{M}(X), \gamma_{D_{M}(X)}\right)$. As a counterexample, consider the self-map $g$ of $\left.\left(D_{M}(X), \gamma_{D_{M}(X)}\right)\right)$ as follows (see Figure $\left.4(b)\right)$ :

$$
g\left(D_{M}(X) \backslash\left\{x_{1}, x_{2}, x_{3}\right\}\right)=\left\{x_{3}\right\}, g\left(x_{3}\right)=x_{0}, g\left(x_{1}\right)=x_{2}, g\left(x_{2}\right)=x_{1} .
$$

Then, whereas the map g of (3.2) is obviously an M-continuous map, it does not have any fixed point, which means that $\left(D_{M}(X), \gamma_{D_{M}(X)}\right)$ does not have the FPP.

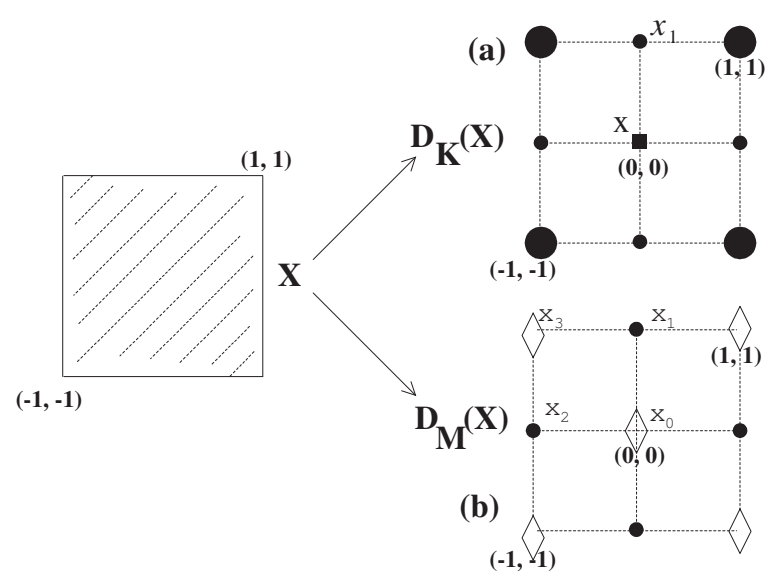

Figure 4: (a) A K-digitization of the plane $X$, denoted by $D_{K}(X) ;(b)$ an M-digitization of the plane $P$, denoted byD ${ }_{M}(X)$, where $X:=[-1,1]^{2} \subset \mathbf{R}^{2}$.

\section{Remarks on the digital homotopy fixed point property}

To study the DHFP of a digital image $(X, k)$, the paper [3] used the following digital k-homotopy.

Definition $4.1([2,5])$. Let $\left(X, k_{0}\right)$ in $Z^{n_{0}}$ and $\left(Y, k_{1}\right)$ in $Z^{n_{1}}$ be digital images. Let $f, g: X \rightarrow Y$ be $\left(k_{0}, k_{1}\right)$ continuous functions. Suppose there exist $\mathrm{m} \in \mathbf{N}$ and a function $\mathrm{H}: X \times[0, \mathrm{~m}]_{\mathbf{Z}} \rightarrow \mathrm{Y}$ such that

- for all $x \in X, H(x, 0)=f(x)$ and $H(x, m)=g(x)$;

- for all $x \in X$, the induced function $H_{x}:[0, m]_{Z} \rightarrow Y$ given by $H_{x}(t):=H(x, t)$ for all $t \in[0, m]_{Z}$ is $\left(2, k_{1}\right)$-continuous; and

- for all $t \in[0, m]_{Z}$, the induced function $H_{t}: X \rightarrow Y$ given by $H_{t}(x):=H(x, t)$ for all $x \in X$ is $\left(k_{0}, k_{1}\right)$-continuous. 
Then we say that $\mathrm{H}$ is a $\left(\mathrm{k}_{0}, \mathrm{k}_{1}\right)$-homotopy between $\mathrm{f}$ and $\mathrm{g}$.

When $f$ and $g$ are $\left(k_{0}, k_{1}\right)$-homotopic in $Y$, we denote by $f \simeq_{\left(k_{0}, k_{1}\right)} g$ the homotopic relation [2]. In addition, if $n_{0}=n_{1}$ and $k_{0}=k_{1}$, then we say that $f$ and $g$ are $k_{0}$-homotopic in $Y$ and use the notation $f \simeq_{k_{0}} g$. If, for some $x_{0} \in X, 1_{X}$ is $k$-homotopic to the constant map $c_{\left\{x_{0}\right\}}$ in the space $\left(X, x_{0}\right)$, then we say that $\left(X, x_{0}\right)$ is k-contractible [2].

In ordinary fixed point theory, Szymik [21] raised the following question: how do the fixed points of a continuous self-map depend on the given self-map? To work this issue out in detail, the author defined the following notions.

Definition 4.2 ([21], Continuous family of self-maps). Let $X$ be a topological space. A continuous family of self-maps of $X$ is a continuous map $f: T \times X \rightarrow X$, where $T$ is another topological space. A continuous family $f: T \times X \rightarrow X$ of self-maps defines for each $t \in T$ a self-map

$$
f_{t}: X \rightarrow X, x \rightarrow f(t, x)
$$

of $X$ which is continuous.

Assume every continuous family of self-maps for a given self-map. Continuous family of fixed points need not exist, even if both $T$ and $X$ satisfy the FPP (see Example 5.3 of the paper [21]). Hence the above approach can be helpful to study topological spaces from the viewpoint of fixed point theory. To establish the DHFP, let us recall the following notions [21] (see Definitions 4.3, 4.4, and 4.5 below).

Definition 4.3 ([21], Continuous family of fixed points). Let $f: T \times X \rightarrow X$ be a continuous family of self-maps of $X$. A continuous family of fixed points of $f$ is a continuous map $p: T \rightarrow X$ such that $f(t, p(t))=p(t)$ for all $t \in T$. The condition (4.1) means that $p(t)$ is a fixed point of $f_{t}$ for all $t \in T$.

Definition 4.4 ([21], Fixed point property with respect to a topological space). A topological space $X$ has the FPP with respect to a topological space $T$ if for all continuous families $f: T \times X \rightarrow X$ of self-maps of $X$, there exists at least one continuous family $p: T \rightarrow X$ of fixed points.

Definition 4.5 ([21]). A topological space $X$ has the HFP if it has the FPP with respect to the unit interval $[0,1]$.

Motivated by these notions, the authors of [3] formulated a digital version of the HFP in terms of a normal adjacency relations of digital products (see Definition 2.2). Using the adjacency of Definition 2.2, we propose the following:

Definition 4.6 ([3]). A digital image $(X, k)$ has the DFP with respect to a digital interval $[0, m]_{Z}$ if for all $\left(k_{*}, k\right)$-continuous maps $f:\left([0, m]_{\mathbf{Z}} \times X, k_{*}\right) \rightarrow(X, k)$, where $k_{*}$ is a normal adjacency for $[0, m]_{Z} \times X$, there exists at least one $(2, k)$-continuous map $p:[0, m]_{Z} \rightarrow X$ of fixed points.

Definition $4.7([3])$. A digital image $(X, k)$ has the DHFP if it has the DFP with respect to $[0, m]_{Z}$ for all integers $m \geqslant 0$.

Definition 4.7 can be stated as follows: a digital image $(X, k)$ has the DHFP if for all digital homotopies $f:[0, m]_{Z} \times X \rightarrow X$ in $X$ there is a $(2, k)$-continuous $k$-path $p:[0, m]_{Z} \rightarrow X$ such that $p(t)$ is a fixed point of $f_{t}$ for all $t \in[0, m]_{z}$. Based on this notion, motivated by the retraction property of HFP in [21] (see Propositions 4.1 and 4.2 of [21]), the paper [3] proposed some results (see Propositions 4.8 and 4.9 below) by using both a k-retraction and the DFP of a digital image $(X, k)$ with respect to a digital interval $[0, \mathrm{~m}]_{\mathrm{Z}}$. Since $[0, n]_{Z}$ is a 2 -retract of $[0, m]_{Z}, n \leqslant m$, we obtain the following:

Proposition 4.8 ([3]). If a digital image $(\mathrm{X}, \mathrm{k})$ has the DFP with respect to a digital interval $[0, \mathrm{~m}]_{\mathrm{Z}}$, then $(\mathrm{X}, \mathrm{k})$ has the DFP with respect to $[0, \mathrm{n}]_{\mathbf{Z}}$, where $\mathrm{n} \lesseqgtr \mathrm{m}$. 
Proposition 4.9 ([3]). Assume that a digital image $(\mathrm{X}, \mathrm{k})$ has the DFP with respect to a digital interval $[0, \mathrm{~m}]_{\mathrm{Z}}$. If $(A, k)$ is a k-retract of $(X, k)$, then $(A, k)$ also has the DFP with respect to $[0, m]_{Z}$.

Corollary 4.10 ([3]). The DHFP requires the DFP. It is clear that the converse need not be valid.

Furthermore, in view of Proposition 4.8, we obtain the following because a digital image $(X, k)$ has the $F P P$ with respective to a singleton if and only if it has the FPP.

Corollary 4.11. The DFP requires the FPP. It is clear that the converse need not be true.

Thus we see that if a given digital image $(X, k)$ does not have the DFP (resp. FPP), then it does not have the DHFP (resp. DFP) either. According to Proposition 1.1 and Corollaries 4.10 and 4.11, we obtain the following:

Theorem 4.12. The digital image $(X, k)$ satisfies the DFP if and only if it is a singleton.

Proof. Since a singleton $(X, k)$ in $\mathbf{Z}^{\mathfrak{n}}$, e.g., $X:=\{x\}$ has the $F P P$, for every integer interval $[0, m]_{Z}$ whenever we consider continuous family of fixed points of a self-map $f$ of $X$ is exactly a $(2, k)$-continuous map, denoted by $p_{t}: X \rightarrow X$ whose graph of $p_{t}$ can be considered as the set $\left\{(t, x) \mid t \in[0, m]_{Z}, x \in X\right\}$. Thus we see that a singleton has the DFP.

Conversely, suppose that there is a digital image $(X, k)$ in $\mathbf{Z}^{\mathrm{n}}$ whose cardinality is greater than 1 and having the DFP. Then, let us examine if for some $[0, \mathrm{~m}]_{\mathbf{Z}}$ we obtain a $(2, \mathrm{k})$-continuous map of fixed points

$$
\mathrm{F}:\left([0, \mathrm{~m}]_{\mathbf{Z}} \times X, \mathrm{k}_{*}\right) \rightarrow(\mathrm{X}, \mathrm{k}) .
$$

In (4.2) we only consider the case $[0, \mathrm{~m}]_{\mathbf{Z}} \times \mathbf{X}$ has the normal $\mathrm{k}_{*}$-adjacency. Indeed, by Proposition 1.1 , we see that only a singleton has the FPP. Thus the digital image $(X, k)$ whose cardinality is greater than 1 cannot have the $(2, \mathrm{k})$-continuous map of fixed points inherited from the homotopy of (4.2) because it does not satisfy the FPP. As a result, according to Proposition 1.1, and Corollaries 4.10 and 4.11, we conclude that the given digital image $(X, k)$ should be a singleton.

To guarantee Corollary 4.11, the authors of [3] proposed the following example.

Example 4.13 ([3, Example 4.1]). Let $X=[a, a+1]_{\mathbf{Z}}$ be a digital interval where $a \in \mathbf{Z}$. It is easy to see that $(\mathrm{X}, 2)$ has both the DFP and the DHFP.

However, the assertion in Example 4.13 is incorrect because the given digital image $(X, 2)$ does have neither the FPP nor the DFP (see Proposition 3.12, Corollaries 4.10 and 4.11 and Theorem 4.12). The below Remark 4.14 and Example 4.15 verify the claim.

Remark 4.14. Unlike the assertion of [3] as in Example 4.13 above, the digital image $(X, 2)$ in Example 4.13 satisfies neither the DFP nor the DHFP. To be specific, assume the self-map

$$
f:[a, a+1]_{\mathbf{Z}} \rightarrow[a, a+1]_{\mathbf{Z}}
$$

given by

$$
f(a)=a+1 \text { and } f(a+1)=a .
$$

Namely, as discussed in Proposition 1.1, it is clear that while the map $f$ is a 2-continuous map, it does not have any fixed point (see also Proposition 1.1). Hence, by Corollaries 4.10, 4.11, and Theorem 4.12, we see that the digital image $(X, 2)$ does not have the DFP because the DHFP (resp. DFP) is stronger than the $D F P$ (resp. FPP in digital topology).

To be specific, we can guarantee Remark 4.14 more precisely with the following example. 
Example 4.15. We see that the given digital image $(X, 2)$ in Example 4.13 does not have the DHFP because the digital image $(X, 2)$ does not have the FPP from the viewpoint of digital topology (see Remark 4.14).

More precisely, let us assume the two digital images $T:=\left([0,3]_{Z}, 2\right)$ and $X:=\left([a, a+1]_{Z}, 2\right)$. According to Definition 2.2, we take a normal 8-adjacency for the digital product $T \times X$, i.e., $(T \times X, 8)$. Indeed, in this case only the 8 -adjacency is normal for the product $T \times X$. Let us now consider the $(8,2)$-continuous families of self-maps (see Figure 2) as follows:

$$
\mathrm{F}:(\mathrm{T} \times \mathrm{X}, 8) \rightarrow(\mathrm{X}, 2) \text { or } \mathrm{f}_{\mathrm{t}}:(\mathrm{X}, 2) \rightarrow(\mathrm{X}, 2), \mathrm{t} \in[0,3]_{\mathrm{Z}}
$$

given by

$$
\left\{\begin{array}{l}
f_{0}=1 x, \\
f_{1}(a)=a+1 \text { and } f_{1}(a+1)=a, \\
f_{2}=C_{\{a\}} \text { as a constant map onto the singleton }\{a\}, \\
f_{3}=C_{\{a+1\}} \text { as a constant map onto the singleton }\{a+1\} .
\end{array}\right\}
$$

Then the fixed point sets of the maps $f_{t}$ are

$$
\left\{x \in X \mid f_{t}(x)=x\right\}=\left\{\begin{array}{ll}
X, & t=0 \\
\emptyset, & t=1 \\
\{a\}, & t=2 \\
\{a+1\}, & t=3
\end{array}\right\}
$$

This property (4.3) makes it clear that there is no 2-continuous family of fixed points. Finally, we prove that Example 4.13 (or Example 1 of the paper [3]) is incorrect.

Theorem 4.16. A digital image $(\mathrm{X}, \mathrm{k})$ has the DHFP, the DFP, and the FPP if and only if it is a singleton.

Proof. In digital topology, by Proposition 1.1 and Corollaries 4.10 and 4.11, since the digital image satisfying the DHFP is only a singleton, the proof is completed.

\section{Non-homotopy invariant of the digital homotopy fixed point property}

The following notion of a digital homotopy equivalence firstly developed in the paper [4] to classify digital images up to a digital k-homotopy equivalence.

Definition $5.1([4])$. Let $f:\left(X, k_{0}\right) \rightarrow\left(Y, k_{1}\right)$ and $g:\left(Y, k_{1}\right) \rightarrow\left(X, k_{0}\right)$ be $\left(k_{0}, k_{1}\right)$ - and $\left(k_{1}, k_{0}\right)$-continuous maps, respectively, such that

$$
g \circ f \simeq_{k_{0}} 1_{X} \text { and } f \circ g \simeq_{k_{1}} 1_{Y} .
$$

Then we say that $\left(X, k_{0}\right)$ and $\left(Y, k_{1}\right)$ have the same $\left(k_{0}, k_{1}\right)$-homotopy type (or $\left(X, k_{0}\right)$ is $\left(k_{0}, k_{1}\right)$-homotopy equivalent to $\left.\left(Y, k_{1}\right)\right)$.

Theorem 5.2. The DHFP is not a digital homotopy equivalence invariant.

Proof. It suffices to give two examples to guarantee this assertion, e.g., $\left(\left[0, l_{\mathbf{Z}}, 2\right)\right.$ and another digital image $(X, k)$ which is $k$-homotopy equivalent to a singleton. Consider a singleton of $[0, l]_{Z}, l \geqslant 1$. It is clear that $\left(\left[0, l_{\mathbf{Z}}, 2\right)\right.$ is 2 -homotopy equivalent to any singleton $\{t\}, t \in[0, l]_{\mathbf{Z}}$ such as $\{0\}$. Then, by Remark 4.14 and Example 4.13 , we proved that $\left[0, l_{\mathbf{Z}}\right.$ does not have the DHFP because $\left(\left[0, l_{\mathbf{Z}}, 2\right)\right.$ does not have the FPP (see Proposition 1.1). But the singleton $\{t\}, t \in\left[0, l_{Z}\right.$ obviously has the DHFP. For convenience, we suffice to prove that the singleton $\{0\}$ has the DHFP. To be specific, let us consider the 2-continuous families of self-maps for any $\mathrm{T}:=[0, \mathrm{~m}]_{\mathbf{Z}}, \mathrm{m} \in \mathbf{N}$ as follows:

$F:(T \times\{0\}, 2) \rightarrow(\{0\}, 2)\left(\right.$ or $\left.f_{t}:(\{0\}, 2) \rightarrow(\{0\}, 2), t \in[0, m]_{z}\right)$ given by

$$
f_{t}=1_{\{0\}}, \quad t \in[0, m]_{Z},
$$


which is the identity map onto the singleton $\{0\}$. Then, according to (5.1), we obtain the fixed point sets of the maps $f_{t}$ as the set $\left\{(t, 0) \mid t \in[0, l]_{\mathbf{Z}}\right\}$. Hence there is a $(2, k)$-(or 2-)continuous map of fixed points whose image can be considered to be the set $\left\{(t, 0) \mid t \in[0, m]_{\mathbf{Z}}\right\}$.

In general, consider any $k$-contractible digital image $(X, k)$ in $Z^{n}$ such that $|X| \geqslant 2$. Then it is clear that it is $k$-homotopy equivalent to any singleton $(\{x\}, k) \subset(X, k)$. While the singleton $(\{x\}, k)$ has the DHFP, the digital image $(X, k)$ does not have the DHFP because $(X, k)$ does not have the FPP (see Proposition 1.1).

In addition, we further need to comment on the contents in Section 4 of the paper [3], such a kind of approach as in [3] is trivial with the following reason:

Remark 5.3. Owing to Corollaries 4.10, 4.11, and Proposition 1.1, it is clear that only a singleton digital image satisfies the DHFP. Hence the digital images, e.g., $(X, 8)$, where $X:=[-1,3]_{Z}^{2} \backslash\{(0,0)\}$ or $S C_{8}^{2,4}$, are far from the study of the digital homotopy fixed point theory because none of $(X, 8)$ and $\mathrm{SC}_{8}^{2,4}$ does not have the FPP.

\section{The digital homotopy almost fixed point property}

In view of Proposition 1.1, we need to expand DHFP into the DHAFP. Rosenfeld [18] firstly studied the AFPP so that not every k-continuous self-map $f$ of a digital image $(\mathrm{X}, \mathrm{k})$ satisfies the AFPP [18].

Let us now generalize the DFP of Definition 4.6 into the following:

Definition 6.1. A digital image $(X, k)$ has the DAFP with respect to a digital interval $[0, m]_{Z}$ if for all $\left(k_{*}, k\right)$ continuous maps $f:\left([0, m]_{\mathbf{Z}} \times X, k_{*}\right) \rightarrow(X, k)$ where $k_{*}$ is a normal adjacency relation for $[0, m]_{\mathbf{Z}} \times X$, there exists at least one $(2, k)$-continuous map $p:[0, m]_{Z} \rightarrow X$ of almost fixed points.

Definition 6.2. A digital image $(\mathrm{X}, \mathrm{k})$ has the DHAFP (resp. DHFP) if it has the DAFP (resp. DFP) with respect to $[0, m]_{Z}$ for all integers $m \geqslant 0$.

In view of Proposition 1.1, we need to study the DHAFP which is broader than the DHFP. Hence, by using the adjacency of Definition 2.2, we propose the following: Definition 6.2 can be represented as follows: a digital image $(X, k)$ has the DHAFP if for all digital homotopies $f:[0, m]_{Z} \times X \rightarrow X$ in $X$ there is a $(2, k)$-continuous $k$-path $p:[0, m]_{Z} \rightarrow X$ such that $p(t)$ is an almost fixed point of $f_{t}$ for all $t \in[0, m]_{Z}$.

We obviously obtain the following.

Proposition 6.3. The DHAFP requires the DAFP. But the converse need not be valid.

Theorem 6.4. Let $(\mathrm{X}, \mathrm{k})$ be a bounded simple $\mathrm{k}-$ path in $\mathbf{Z}^{\mathrm{n}}, \mathrm{n} \in \mathbf{N}$. Then it has the DHAFP.

Proof. It is clear that a bounded simple $k$-path $\left(X:=\left\{x_{i} \mid i \in[0, t]_{Z}\right\}, k\right)$ in $Z^{n}$ is $(k, 2)$-isomorphic to $\left([0, t]_{\mathbf{Z}}, 2\right)$. Since $\left([0, \mathrm{t}]_{\mathbf{Z}}, 2\right)$ has the AFPP [18] and further, the AFPP is a digital topological invariant, we obtain that $(\mathrm{X}, \mathrm{k})$ has the AFPP. Under this situation, for every integral interval $[0, \mathrm{~m}]_{\mathrm{Z}}$ whenever we consider continuous family of almost fixed points of a self-map $f$ of $X$ is exactly a $(2, k)$-continuous map, denoted by $p_{t}: X \rightarrow X$ whose graph of $p_{t}$ can be considered as the set $\left\{(t, x) \mid t \in[0, m]_{z}, x \in X\right\}$. Thus we obtain that $(\mathrm{X}, \mathrm{k})$ has the DHAFP.

Example 6.5. The integral interval $\left(X:=[a, a+1]_{Z}, 2\right)$ in Remark 4.14 has the DHAFP. To be specific, consider any 2-continuous self-map $f$ of $[a, a+1]_{Z}$. Then it is clear that the digital image $(X, 2)$ has the $A F P P$ and further, the DHAFP (Proposition 6.3). 


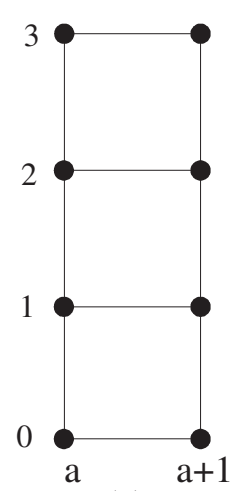

(a)

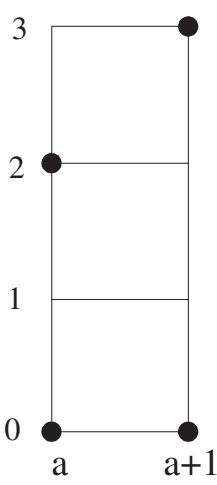

(b)

Figure 5: Explanation of the non-DHFP of $X:=\left([a, a+1]_{\mathbf{Z}}, 2\right)$. (a) $(T \times X, 8) ;(b)$ Graph of $f_{t}, t \in[0,3]_{Z}$.

Theorem 6.6. Let $\mathbf{X}$ be a bounded digital plane in $\mathbf{Z}^{2}$ with 8-adjacency. Then it has the DHAFP.

Proof. It is clear that a bounded digital plane $(\mathrm{X}, 8)$ in $\mathbf{Z}^{2}$ has the AFPP [18]. Under this situation, for every integral interval $[0, m]_{Z}$ whenever we consider continuous family of almost fixed points of a self-map $f$ of $X$ is exactly a $(2,8)$-continuous map, denoted by $p_{t}: X \rightarrow X$ whose graph of $p_{t}$ can be considered as the set $\left\{(t, x) \mid t \in[0, m]_{Z}, x \in X\right\}$. Thus we see that $(X, 8)$ has the DHAFP.

Remark 6.7. In Theorem 6.4, if the given adjacency is not an 8-adjacency, the assertion does not hold.

Proof. Let us consider the set $X:=[0,1]_{\mathbf{Z}} \times[0,1]_{\mathbf{Z}}$ in $\mathbf{Z}^{2}$. Suppose a 4 -adjacency for $X$. Then we see that $(X, 4)$ is a kind of $S_{4}^{2,4}:=\left\{x_{0}=(0,0), x_{1}=(1,0), x_{2}=(1,1), x_{3}=(0,1)\right\}$. Then it is clear that $(X, 4)$ does not have the AFPP. To be specific, consider the self-map of $(X, 4)$ given by $f\left(x_{i}\right)=x_{i+2(\bmod 4)}$. While the map $\mathrm{f}$ is a 4-continuous map, it does not have the AFPP. By Proposition 6.3, the proof is completed.

By using the method similar to Theorem 6.4, we obtain the following:

Corollary 6.8. Let $\mathrm{X}$ be a bounded digital cube $\mathrm{X} \subset \mathbf{Z}^{\mathrm{n}}$ with $\left(3^{\mathrm{n}}-1\right)$-adjacency. Then $\left(\mathrm{X}, 3^{\mathrm{n}}-1\right)$ has the DHAFP.

\section{Concluding remarks}

We have studied a digital version of the HFP for studying the FPP of digital images. Besides, we have proved that the DHFP is not a digital homotopy equivalence invariant. Finally, according to Proposition 1.1, since only a singleton has the FPP from the viewpoint of digital topology followed from Rosenfeld model, it turns out that a digital image satisfies each of the DHFP, the DFP, and the FPP if and only if it is a singleton.

\section{Acknowledgment}

Author was supported by Basic Science Research Program through the National Research Foundation of Korea (NRF) funded by the Ministry of Education, Science and Technology (2016R1D1A3A03918403).

\section{References}

[1] P. Alexandroff, Diskrete räume, Mat. Sb. (N.S.), 2 (1937), 501-518. 3

[2] L. Boxer, A classical construction for the digital fundamental group, J. Math. Imaging Vision, 10 (1999), 51-62. 1, 2.4, $4.1,4$

[3] O. Ege, I. Karaca, Digital homotopy fixed point theory, C. R. Math. Acad. Sci. Paris, 353 (2015), 1029-1033. 1, 1, 4, 4, $4.6,4.7,4,4.8,4.9,4.10,4,4.13,4.14,4.15,5$ 
[4] S.-E. Han, On the classification of the digital images up to a digital homotopy equivalence, J. Comput. Commun. Res., 10 (2000), 194-207. 1, 5, 5.1

[5] S.-E. Han, Non-product property of the digital fundamental group, Inform. Sci., 171 (2005), 73-91. 2, 2, 2, 2, 2.2, $2.3,4.1$

[6] S.-E. Han, Fixed point theorems for digital images, Honam Math. J., 37 (2015), 595-608. 1

[7] S.-E. Han, Contractibility and fixed point property: the case of Khalimsky topological spaces, Fixed Point Theory Appl., 2016 (2016), 20 pages. 1, 3, 3

[8] S.-E. Han, Almost fixed point property for digital spaces associated with Marcus-Wyse topological spaces, J. Nonlinear Sci. Appl., 10 (2017), 34-47. 3, 3.1, 3.2

[9] S.-E. Han, B. G. Park, Digital graph $\left(\mathrm{k}_{0}, \mathrm{k}_{1}\right)$-isomorphism and its applications, Summer conference on topology and its application, USA, (2003). 2.4

[10] G. T. Herman, Oriented surfaces in digital spaces, CVGIP: Graph. Model. Im. Proc., 55 (1993), 381-396. 1

[11] J. M. Kang, S.-E. Han, K. C. Min, Digitizations associated with several types of digital topological approaches, Comput. Appl. Math., 36 (2017), 571-597. 1, 3, 3, 3, 3.3, 3.5, 3.6, 3, 3.7, 1

[12] E. D. Khalimsky, Applications of connected ordered topological spaces in topology, Conference of Mathematics Departments of Povolsia, (1970). 1, 3

[13] E. Khalimsky, R. Kopperman, P. R. Meyer, Computer graphics and connected topologies on finite ordered sets, Topology Appl., 36 (1990), 1-17. 2, 3

[14] T. Y. Kong, A. Rosenfeld, Topological algorithms for digital image processing, Elsevier Science, Amsterdam, (1996). 1

[15] V. Kovalevsky, Axiomatic digital topology, J. Math. Imaging Vision, 26 (2006), 41-58. 1

[16] J. R. Munkres, Topology, Second edition, Prentice Hall, NJ, (2000). 3, 3

[17] A. Rosenfeld, Digital topology, Amer. Math. Monthly, 86 (1979), 76-87. 1, 2, 2

[18] A. Rosenfeld, Continuous functions on digital pictures, Pattern Recognit. Lett., 4 (1986), 177-184. 1, 2, 2, 2, 6, 6, 6

[19] S. Samieinia, The number of Khalimsky-continuous functions between two points, Combinatorial image analysis, Lecture Notes in Comput. Sci., Springer, Heidelberg, 6636 (2011), 96-106. 3

[20] J. Šlapal, Topological structuring of the digital plane, Discrete Math. Theor. Comput. Sci., 15 (2013), 165-176. 3

[21] M. Szymik, Homotopies and the universal fixed point property, Order, 32 (2015), 301-311. 1, 4, 4.2, 4, 4.3, 4.4, 4.5, 4

[22] F. Wyse, D. Marcus, Solution to problem 5712, Amer. Math. Monthly, 77 (1970), 1119. 1, 3, 3 